\title{
Information Entropy and Urban Population: A Case Study of Taipei City
}

\author{
Tian-Yow Shyu \\ Tai-Hwa Hor \\ Lunghwa University of Science and Technology \\ Hsin-Chien Huang \\ Chinese Culture University
}

\begin{abstract}
A city can be regarded as a complex system possessing many functions, including business, industry, culture, education, and so on. Citizens in a city may shift houses due to some significant reasons and various activities which have been studied by different organizations. Sometimes the population moves to a certain area (district), and it makes the population distribution more homogenous. How to convert the qualitative description of population change into a quantitative measurement is the aim of this study. Here we apply dada analysis concept to proceed to the urban population system. The total number of population in Taipei City changes every year, and based upon the population data bank, we try to calculate its information entropy. The primary results show that the variation of the information entropy indeed matches with the mechanism of the city's population movement. Moreover, the activities of human society resemble statistically dynamic phenomena in that the macro system is an ensemble of the micro systems.
\end{abstract}

Keywords: Information entropy, urban population, Taipei City

\section{Introduction}

In physics, entropy is a measurement of work ability in a system. It also plays an important role in many fields. Schrodinger (1944) used the concept of entropy to explain the life phenomena and showed that life greatly depends on order, and a living organism has to consist of a large number of atoms with ordered assemble. Shannon (1949) used the similar concept of entropy to study the information theory. Wilson (1970) used the concept of entropy to research social problems of a city. Alam and Williams (1993) used cross entropy to measure dissimilarity between populations. Esmer (2005) used information entropy to study urban spatial structure. Kazimieras (2020) used Shannon Entropy Index to assess the progress in strategic priorities among EU countries. In statistical thermodynamics, the behavior of a macro system depends on the probability of physical phenomena of its micro systems. In information theory, information entropy is a measurement of unpredictability of information content. In nature, the change of entropy in a closed system is always greater than zero. If the change of entropy is less than zero, some work has to be done on the system. In information theory, if the information comes to a homogeneous and disordered state, the information entropy has the greatest value. If the information becomes predictable or ordered, the value of information entropy is relatively small. A city can be considered as a system, and the population of the city can be taken as a source of information. The change of population is also a change of informationandthe movement of popultion induces many nonlinear effects. In reality, the population of Taipei City changes every year. It increases in some years while decreasing in some other years. Also, some citizens move from one district to another at the same time. Because a city is an open system in which people, materials and resources flow in and out very frequently, the distribution of population becomes ordered at sometime, while disordered at some other time. In this study, we use information entropy to investigate the population variation with quantitative computation of people's movement from one district (as a micro system) to the other one in Taipei City (as a macro system).

\section{Data and Methodology}

A city is a complex system, and the districts in the city possess characteristic features in business, industry, culture, education, etc. In Taipei City, there are 12 districts, namely Songshan, Xinyi, Da'an, Zhongshan, Zhongzheng, Datong, Wanhua, Wenshan, Nankang, Neihu, Shilin, and Beitou, as shown in Fig.1. The total population of Taipei City from 1990 to 2012 is shown in Fig.2. It can be seen that the population varies from year to year with the maximum magnitude of 2,719,659 in 1990. Because the districts have different progress and infrastructure, it makes the population movement from one district to another very frequently. Therefore, residents intend to move toward one certain district due to urban renewal, while at some other time the distribution of population appears homogeneous. Also, people will move out of the city due to the jump of house price. 
Consequently, the distribution of population in a city contains a lot of information. In this respect, the information entropy can be used to express the order or disorder of population distribution.

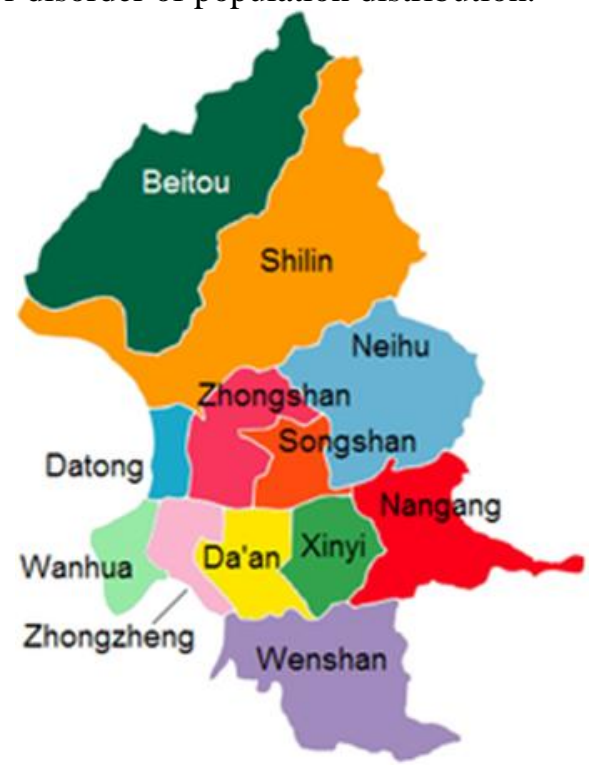

Fig.1 Map of Taipei Citywhich covers 12 districts.

According to Boltzmann's theory, the entropy is represented by $S=k_{\mathrm{B}} \ln W$, and the entropy change is $\mathrm{d} S=S_{\mathrm{f}}-S_{\mathrm{i}}$, where $k_{\mathrm{B}}$ is the Boltzmann constant, $W$ is the probability of particles and $\mathrm{d} S$ is the entropy change from initial state $S_{\mathrm{i}}$ to final state $S_{\mathrm{f}}$. If the system is an open one, and the entropy of the system decreases (increases), i.e.dS $<0(\mathrm{dS}>0)$, then the system proceeds toward ordered (disordered) direction, or some work must be done on (by) the system. On the other hand, $\mathrm{d} S=0$ indicates that the entropy flows in and out of the system are equal. In this context, every resident in the city can be viewed as a particle.

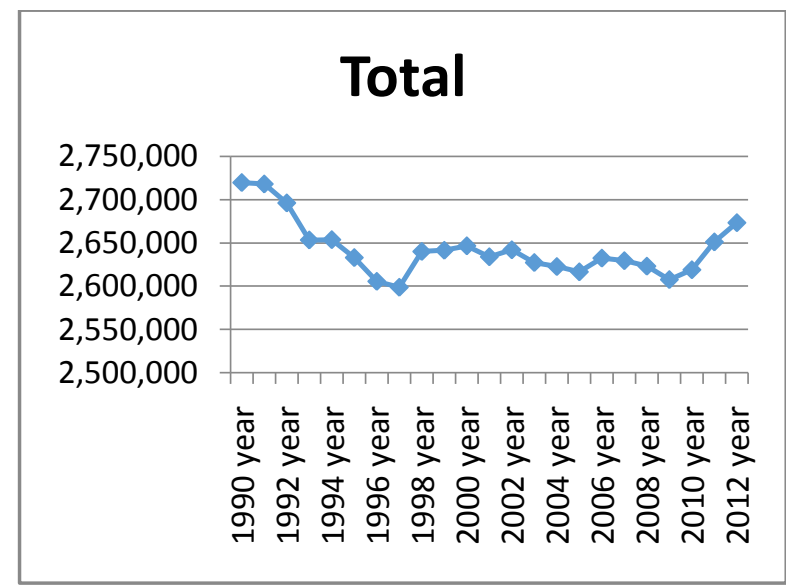

Fig.2 The population of Taipei City from 1990 to 2012.

Here, we use information entropy to quantify the information of Taipei City. The Shannon information entropy $H$ is given by the following formula (1):

$$
H=-\sum p_{i} \log _{b} p_{i,}(1)
$$

Where $H$ stands for the information entropy of Taipei City in any year, $p_{i}$ represents the probability of population in 12 different districts of Taipei City and $p_{i}=$ (the population of individual district of Taipei City)/(the total population of Taipei City).

Fig.3shows the information entropy of Taipei City from 1990 to 2012. Startingin1996, the information entropy decreased continuously till 2009 , and then, it turns to increase. 


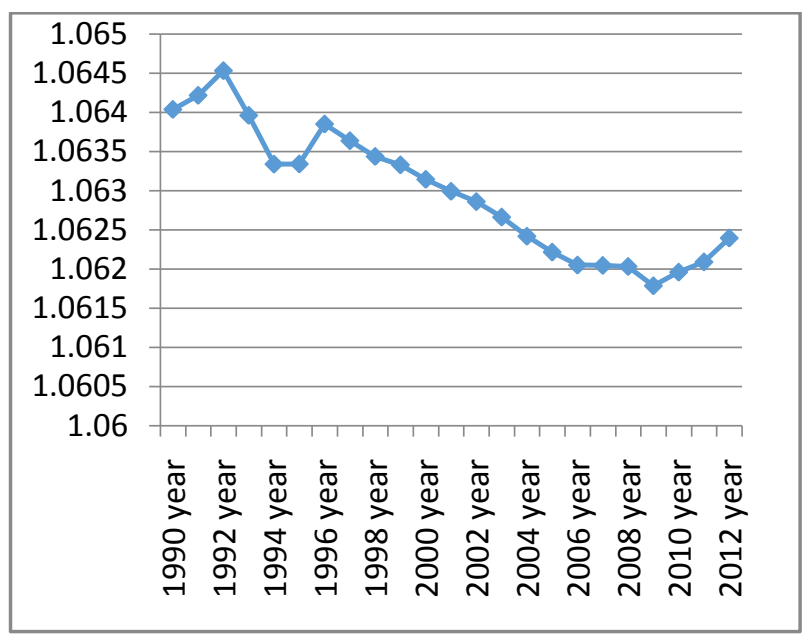

Fig.3 The information entropy of Taipei City from 1990 to 2012.

In Fig.4, the blue line is the total population of Taipei City while the black line is the tendency of population change from 1998 to 2009. It clearly indicates the decline of population. At the same time, it can be seen from Fig. 3that the entropy also decreased. The decline of population matches with entropy decrease. But after 2009, both the population and information entropy increased simultaneously. The reasons will be discussed later.

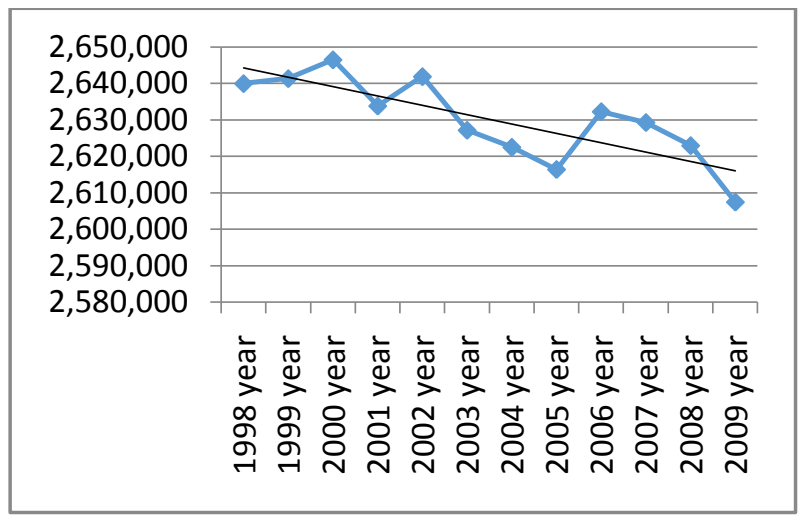

Fig.4 The population (bluecurve) and variation trend (black curve) of Taipei City from 1998 to 2009.

\section{Analysis and Discussion}

It can be seen fromFig.4 that the information entropy changes every year. The information entropy has the same meaning as the Boltzmann thermodynamic entropy. If the information entropy changed, some people were also possible to move toward certain district and vice versa. As a city is overloaded during city development, people may feel uncomfortable and leave the crowded area. This makes the distribution of population more homogeneous and the entropy increases, as can be seen from 1990 to 1993 in Figs.2 and 3.

Figs.5, 6, and 7show the population of Neihu, Wanhua, and Zongshan Districts from 1990 to 2012. It shows that the population increases gradually because Neihu District is a developing industrial park. However, Wanhua District is an old business area and the population decreases steadily. Zongshan District contains both new and old business areas, and new construction and businesses activities due to the urban development of city cause the population from a trend of reduction toward increase. This reveals that the mechanisms of people shifts in different districts are driven differently. In general, wise people will choose fine environments to live. In Fig.3, the information entropy decreased from 1996 to 2009, indicating that some people shifted to other districts or even moved out of Taipei City, making the system more ordered. 


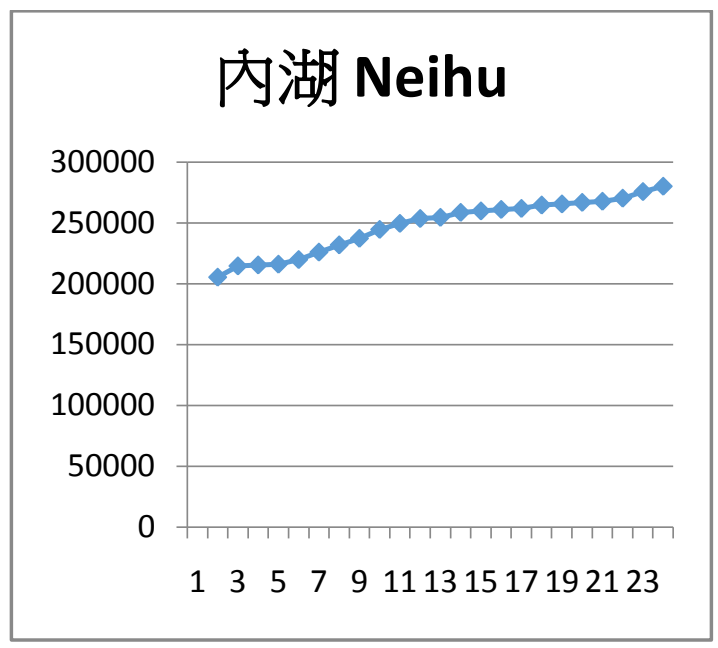

Fig.5 The population of NeihuDistrict from 1990 to 2012.

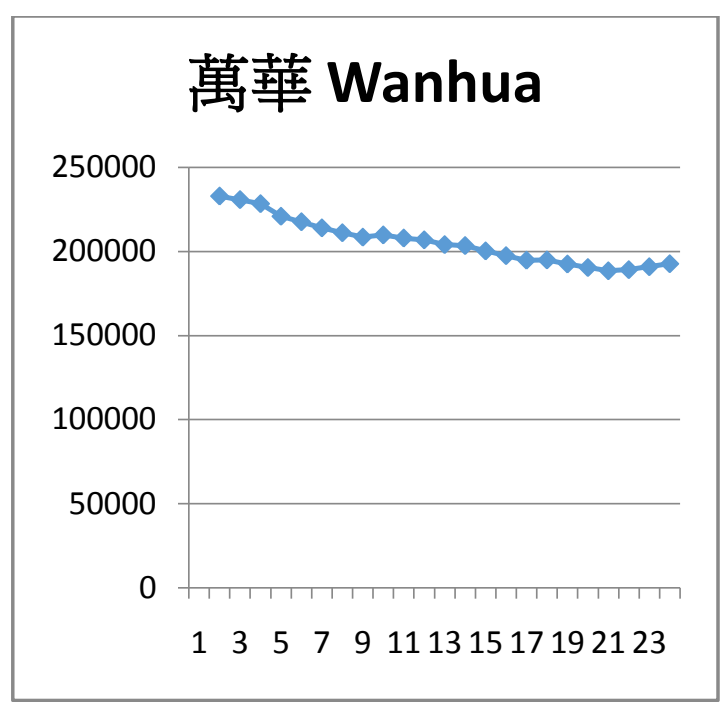

Fig.6 The population of WanhuaDistrictfrom 1990 to 2012.

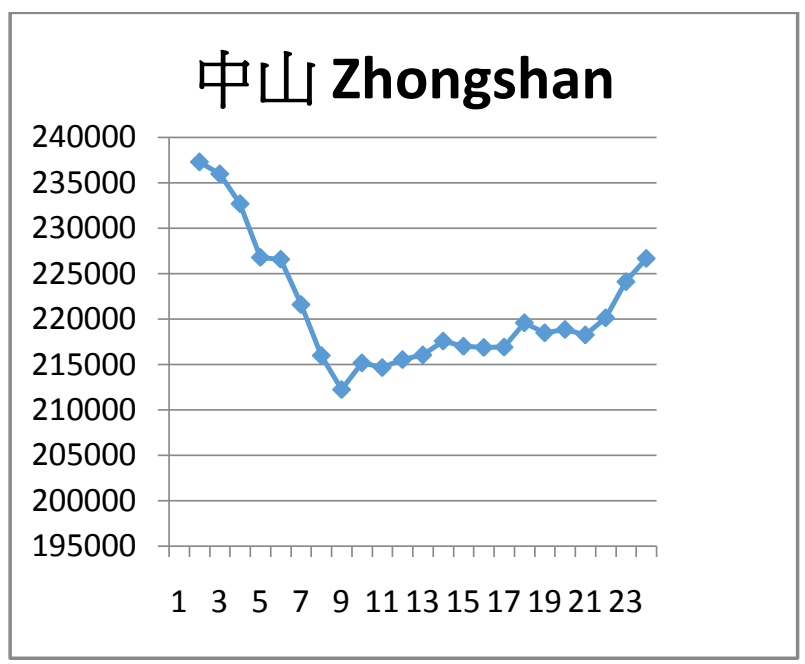

Fig.7 The population of Zhongshan District from 1990 to 2012. 


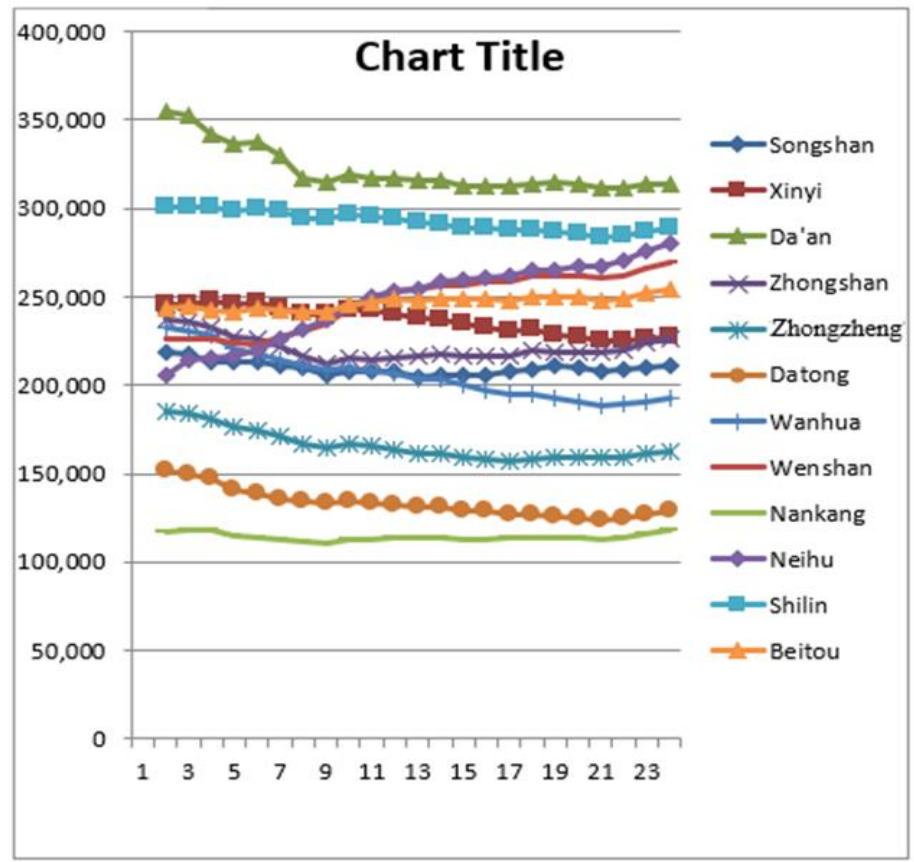

Fig.8 The population of 12 districts of Taipei City from 1990 to 2012.

In 1996, the mass rapid transportation (MRT) system in Taipei City started its operation, and it indicates that the tendency of population changes of Taipei City varied from decreasing to increasing, especially in those districts where the MRT passed through. After 2009, the infrastructure of the whole Taipei City was obviously improved and the entire city population, including 12districts, also increased in recent years, as shown in Fig.8. This caused the entropy increase simultaneously in recent years, as revealed in Fig.3.

\section{Conclusions}

The population of a city is constantly changing because the functionsand living standards of a city vary with time, and wise people will choose fine environments to live. Since the population is one of the significant information of a city, the movement of city residents will be directly related to this factor. If the infrastructure and public facilities for certain area in the city have been improved, it means that there is positive entropy impact to this area, or work must be done by it. Therefore, the distribution of population becomes more homogenous due to the increase of information entropy. In this study, we utilized the information entropy to measure the population variation quantitatively and found that it matches the mechanism of population movement. The results also showed that the phenomenon of a macroscopic social system is influenced by the microscopic individual systems. It is similar to the statistic mechanics, mentioning thatif the micro systems become more homogenous, the macro system will be disordered, and vice vesa.That shows that from our study the information entropy can play a good role to measure the population variation alternatively.

\section{References}

Schrodinger, E. (1944).What is life? Cambridge University Press, Cambridge, MA.

Shannon, C. (1949).Communication Theory of Secrecy Systems. Bell System Technical Journal. 28 (4), 656-715.

Wilson, A. G. (1970).Entropy in urban and regional modeling. Pion, London.

Alam, K., \& Williams, C. L.(1993).Relative difference in diversity between populations. Ann. Inst. Statist. Math.45(2), 383-399.

Esmer, Ö. (2005).Information theory, entropy and urban spatial structure. PhD thesis, Middle East Technical University.

Kazimieras, B. E.(2020).Assessment of progress towards "Europe 2020" strategy targets by using the Multimoora method and the Shannon Entropy Index, Journal of Cleaner Production, Volume 244, 20 January 2020, Article 118895 . 